\title{
REPRESENTATION OF CONTINUOUS FUNCTIONS AS SUMS OF GREEN FUNCTIONS
}

\author{
STEPHEN J. GARDINER
}

(Communicated by Albert Baernstein II)

\begin{abstract}
Let $K \subset \Omega \subseteq \mathbb{R}^{n}$, where $K$ is polar and compact and $\Omega$ is a domain with Green function $G_{\Omega}(\cdot, \cdot)$. We characterize those subsets $E$ of $\Omega \backslash K$ which have the following property: Every positive continuous function on $K$ can be written as $\sum_{k} \lambda_{k} G_{\Omega}\left(x_{k}, \cdot\right)$, where $x_{k} \in E$ and $\lambda_{k}>0$ for each $k$.
\end{abstract}

\section{INTRODUCTION}

Let $P(\cdot, \xi)$ denote the Poisson kernel for the unit disc $D$ with pole $\xi$ in $\partial D$. Hayman and Lyons [11] have characterized those subsets $E$ of $D$ for which every (strictly) positive continuous function $f$ on $\partial D$ can be represented as $f=$ $\sum_{k} \lambda_{k} P\left(z_{k}, \cdot\right)$, where $z_{k} \in E$ and $\lambda_{k}>0$ for each $k$. The convergence here is uniform on $\partial D$, by Dini's theorem. Related results and generalizations may be found in $[2,3,7,8,9]$. Hayman $[10, \S 2.4]$ subsequently asked about the possibility of obtaining a similar representation for continuous functions defined on other compact sets. In this paper we provide a representation of this type for positive continuous functions defined on an arbitrary compact polar set $K$ in $\mathbb{R}^{n}(n \geq 2)$. Green functions, rather than Poisson kernels, are now the building blocks. In particular, when $n \geq 3$, the Newtonian kernels $|x-\cdot|^{2-n}$ may be used, where $|\cdot|$ denotes the Euclidean norm on $\mathbb{R}^{n}$. The restriction to polar sets may be circumvented by identifying functions on a given compact set $K$ with functions on $K \times\{0\}^{2}$, which is a polar set in $\mathbb{R}^{n+2}$.

A domain (nonempty connected open set) $\Omega$ in $\mathbb{R}^{n}$ is called Greenian if it possesses a Green function $G_{\Omega}(\cdot, \cdot)$. When $n \geq 3$ all domains are Greenian; when $n=2$ a domain $\Omega$ is Greenian if and only if $\mathbb{R}^{2} \backslash \Omega$ is not a polar set (Myrberg's theorem). If $K$ is a compact set, then we denote by $C^{+}(K)$ the collection of all (strictly) positive continuous functions on $K$. We state below a special case of a result of Wallin [15] which shows that, if we wish to represent functions in $C^{+}(K)$ as a sum of the form $\sum_{k} \lambda_{k} G_{\Omega}\left(x_{k}, \cdot\right)$, where $\lambda_{k}>0$ for each $k$, then we should consider only polar compact sets $K$. A simple proof is given in $\S \S 3.2,3.3$ for the reader's convenience.

Proposition 1. Let $K$ be a compact set in $\mathbb{R}^{n}$ and let $\Omega$ be a Greenian domain which contains $K$. The following are equivalent:

Received by the editors June 14, 1994 and, in revised form, October 4, 1994

1991 Mathematics Subject Classification. Primary 31B05. 
(i) every member of $C^{+}(K)$ has a positive superharmonic extension to $\Omega$;

(ii) $K$ is polar.

If $K$ is a compact set, then we define $d_{K}(x)=\inf \{|x-y|: y \in K\}$ for each $x$ in $\mathbb{R}^{n}$. Given any subset $E$ of $\mathbb{R}^{n} \backslash K$ and any positive number $\rho$, we define $E_{\rho}=\bigcup_{x \in E} B\left(x, \rho d_{K}(x)\right)$, where $B(x, r)$ is the open ball of centre $x$ and radius $r$. Our main theorem below involves the notion of thinness which is discussed briefly in $\S 2$.

Theorem 1. Let $K$ be a polar compact set in $\mathbb{R}^{n}$ with more than one point, let $\Omega$ be a Greenian domain which contains $K$, and let $E \subseteq \Omega \backslash K$. The following are equivalent:

(a) for each $f$ in $C^{+}(K)$ there exist a sequence $\left(x_{k}\right)$ of points in $E$ and a sequence $\left(\lambda_{k}\right)$ of positive numbers such that

$$
f=\sum_{k} \lambda_{k} G_{\Omega}\left(x_{k}, \cdot\right) \quad \text { on } K
$$

(b) for each $f$ in $C^{+}(K)$ and each positive number $\varepsilon$ there exist sequences $\left(x_{k}\right)$ and $\left(\lambda_{k}\right)$ as in (a) with the additional property that $\varepsilon>d_{K}\left(x_{k}\right) \rightarrow 0$;

(c) there exists $\rho$ in $(0,1)$ such that $E_{\rho}$ is nonthin at each point of $K$.

Thus, in particular, Theorem 1 implicitly identifies those open subsets of $\Omega \backslash K$ on which the positive superharmonic extensions of Proposition 1(i) can be arranged to be harmonic. Again we note that the convergence in (1) is uniform on $K$. Clearly the implication "(a) $\Rightarrow$ (c)" fails when $K$ contains only one point. In connection with (c) we remark that, if $z$ is an isolated point of $K$ and $0<\rho<1$, then $E_{\rho}$ is nonthin at $z$ if and only if $z \in \bar{E}$. This follows easily from Wiener's criterion.

If we impose certain geometric constraints on $K$, then the nonthinness condition in Theorem 1 can be replaced by a purely metric condition, as the following result shows.

Corollary 1. Let $1 \leq m \leq n-3$, let $K$ be a compact $m$-dimensional Lipschitz manifold in $\mathbb{R}^{n}$, and let $E \subseteq \mathbb{R}^{n} \backslash K$. The following are equivalent:

(a) for each $f$ in $C^{+}(K)$ there exist a sequence $\left(x_{k}\right)$ of points in $E$ and a sequence $\left(\lambda_{k}\right)$ of positive numbers such that

$$
f(z)=\sum_{k} \lambda_{k}\left|x_{k}-z\right|^{2-n} \quad(z \in K)
$$

(b) there exists $\rho$ in $(0,1)$ such that

$$
\int_{\left\{x \in E_{\rho}: d_{K}(x)<1\right\}} d_{K}(x)^{-2}|x-z|^{2-n} d x=+\infty \quad(z \in K) .
$$

Theorem 1 is proved in $\S 4$. In $\S 5$ we observe that Corollary 1 follows easily from Theorem 1 and work of Aikawa [1] concerning quasiadditivity properties of capacity.

\section{REDUCED FUnCTIONS AND THIN SETS}

Let $\Omega$ be a Greenian domain. If $u$ is a nonnegative superharmonic function on $\Omega$ and $A \subseteq \Omega$, then the reduced function of $u$ relative to $A$ in $\Omega$ is defined by

$$
R_{u}^{A}(x)=\inf \{v(x): v \text { is superharmonic on } \Omega,
$$

$$
v \geq 0 \text { on } \Omega, v \geq u \text { on } A\} \quad(x \in \Omega) .
$$

Its lower semicontinuous regularization $\widehat{R}_{u}^{A}$ is superharmonic on $\Omega$. Clearly $0 \leq$ 
$\widehat{R}_{u}^{A} \leq u$. If $u$ is a potential on $\Omega$, or if $A$ is relatively compact in $\Omega$, then $\widehat{R}_{u}^{A}$ is a potential on $\Omega$. We shall use the fact that, if $(A(k))$ is an increasing sequence of subsets of $\Omega$ with union $A$, then $\widehat{R}_{u}^{A(k)} \uparrow \widehat{R}_{u}^{A}$ (see $[6,1$.VI.3(e)]).

The fine topology is the coarsest topology on $\mathbb{R}^{n}$ which makes continuous every superharmonic function on $\mathbb{R}^{n}$. It is strictly finer than the Euclidean topology. A set $A$ in $\mathbb{R}^{n}$ is said to be thin at a point $x$ if $x$ is not a fine limit point of $A$. A polar set is thin everywhere. We will make use of the following characterization of thin sets (see $[6,1 . \mathrm{XI}]$ for this and other material concerning the fine topology).

Theorem A. Let $\Omega$ be a Greenian domain, let $x \in \Omega$, and let $A \subseteq \Omega$. The following are equivalent:

(a) $A$ is thin at $x$;

(b) $\widehat{R}_{G_{\Omega}(x, \cdot)}^{A} \neq G_{\Omega}(x, \cdot)$;

(c) the Riesz measure associated with the potential $\widehat{R}_{G_{\Omega}(x, \cdot)}^{A}$ does not charge $\{x\}$.

\section{Proof of Proposition 1}

3.1. We begin with an elementary lemma which is a variant of [3, Lemma 11] and [11, Theorem 1]. It is formulated with more than one application in mind.

Lemma 1. Let $K$ be a compact Hausdorff space and let $\left(\mathcal{F}_{m}\right)$ be a decreasing sequence of subsets of $C^{+}(K)$. Suppose that, for each $f$ in $C^{+}(K)$, each positive number $\varepsilon$, and each $m$ in $\mathbb{N}$, there exist functions $g_{1}, g_{2}, \ldots, g_{l}$ in $\mathcal{F}_{m}$ and positive numbers $\lambda_{1}, \lambda_{2}, \ldots, \lambda_{l}$ such that

$$
\left|f-\sum_{k=1}^{l} \lambda_{k} g_{k}\right|<\varepsilon \quad \text { on } K .
$$

Then, for each $f$ in $C^{+}(K)$ and each $i$ in $\mathbb{N}$, there exist a sequence $\left(g_{k}\right)$ in $\mathcal{F}_{i}$ and a sequence $\left(\lambda_{k}\right)$ of positive numbers such that

$$
f=\sum_{k=1}^{\infty} \lambda_{k} g_{k}
$$

and such that each $\mathcal{F}_{m}$ contains all but finitely many of the functions $g_{k}$.

To see this, let $f \in C^{+}(K)$ and $i \in \mathbb{N}$. We define $f_{0}=a f$, where $a$ is a number chosen large enough to ensure that $f_{0}>1$ on $K$, and proceed inductively as follows. Given $f_{j}$, where $j \geq 0$, there exist functions $g_{j, 1}, g_{j, 2}, \ldots, g_{j, l(j)}$ in $\mathcal{F}_{i+j}$ and positive numbers $\lambda_{j, 1}, \lambda_{j, 2}, \ldots, \lambda_{j, l(j)}$ such that

$$
\left|\left(f_{j}-2^{-j}\right)-u_{j}\right|<2^{-j-1}
$$

where

$$
u_{j}=\sum_{k=1}^{l(j)} \lambda_{j, k} g_{j, k},
$$

and we define $f_{j+1}=f_{j}-u_{j}$. Clearly $f_{j} \rightarrow 0$. Thus, if we define

$$
u=a^{-1} \sum_{j=0}^{\infty} u_{j}=a^{-1} \sum_{j=0}^{\infty} \sum_{k=1}^{l(j)} \lambda_{j, k} g_{j, k},
$$

we see that $u=a^{-1} f_{0}=f$, and the lemma is proved. 
3.2. To prove that (ii) implies (i) in Proposition 1, suppose that (ii) holds, let $f \in C^{+}(K)$ and $0<\varepsilon<\inf _{K} f$. Since $K$ is polar, $\mathbb{R}^{n} \backslash K$ is nonthin at each point of $K$. It follows from a classical approximation theorem (see [12] or [4] or [5]) that there is a harmonic function $h$ on a neighbourhood $W$ of $K$ such that $|h-f|<\varepsilon$ on $K$. Since $h>0$ on $K$, we can choose a bounded open set $V$ such that $K \subset V, \bar{V} \subset \Omega \cap W$, and $h>0$ on $\bar{V}$. Since $K$ is polar, there is a potential $v$ on $\Omega$ such that $v=+\infty$ on $K$. Further, it can be arranged that $v$ is harmonic on $\Omega \backslash K$ (see, for example, [13, p. 181]). We now choose a positive number $a$ small enough so that $a v<h$ on $\partial V$ and define

$$
w(x)= \begin{cases}\min \{a v(x), h(x)\} & (x \in V), \\ a v(x) & (x \in \Omega \backslash V) .\end{cases}
$$

Clearly $w$ is positive and superharmonic on $\Omega$, and $w=h$ on $K$, so $|w-f|<\varepsilon$ on $K$. It now follows from Lemma 1 (with $\mathcal{F}_{m}$ equal to the class of positive superharmonic functions on $\Omega$ which are continuous on $K$, for each $m$ ) that $f$ can be written as $\sum_{k} w_{k}$, where each $w_{k}$ is a positive superharmonic function on $\Omega$ and continuous on $K$. If we define $u=\sum_{k} w_{k}$, then $u$ is a positive superharmonic extension of $f$ to $\Omega$. Hence (i) holds.

3.3. Conversely, suppose that $K$ is nonpolar and let $z_{0} \in K$. Then there exists a positive number $\eta$ such that the set $K^{\prime}=K \backslash B\left(z_{0}, \eta\right)$ is also nonpolar. Hence, if we define $a=\widehat{R}_{1}^{K^{\prime}}\left(z_{0}\right)$ (reduction relative to $\Omega$ ), we see that $a>0$. Now let $f$ be a positive continuous function on $K$ valued 1 on $K^{\prime}$ and $a / 2$ at $z_{0}$. If $u$ is a positive superharmonic extension of $f$ to $\Omega$, then

$$
u\left(z_{0}\right) \geq \widehat{R}_{u}^{K^{\prime}}\left(z_{0}\right)=\widehat{R}_{1}^{K^{\prime}}\left(z_{0}\right)=a>f\left(z_{0}\right),
$$

which yields a contradiction. Hence (i) implies (ii). This completes the proof of Proposition 1.

\section{Proof of Theorem 1}

4.1. In this section we will prove that (c) implies (b) in Theorem 1. First we assemble some preparatory material. We note from Harnack's inequalities that, if $h$ is a positive harmonic function on $B(y, r)$ and $0<\rho<1$, then

$$
\frac{1-\rho^{2}}{(1+\rho)^{n}} \leq \frac{h(x)}{h(y)} \leq \frac{1-\rho^{2}}{(1-\rho)^{n}} \quad(x \in B(y, \rho r)) .
$$

Next we observe below that the value of $\rho$ in condition (c) of Theorem 1 is irrelevant.

Lemma 2. Let $z \in K$, let $E \subseteq \mathbb{R}^{n} \backslash K$, and let $\kappa \in(0,1)$. If $E_{\kappa}$ is thin at $z$, then $E_{\rho}$ is thin at $z$ for all $\rho$ in $(0,1)$.

In the proof of this lemma we may assume that $d_{K}(x) \leq 1$ for all $x$ in $E$, since thinness is a local property. Let $B$ be an open ball containing the set $\left\{x: d_{K}(x) \leq\right.$ $4\}$ and let $l_{n}$ denote the volume of $B(0,1)$. Suppose that $E_{\kappa}$ is thin at $z$, and let $u$ be the regularized reduced function of $G_{B}(z, \cdot)$ relative to $E_{\kappa}$ in $B$. Then $u=G_{B}(z, \cdot)$ on the open set $E_{\kappa}$, but the Riesz measure associated with $u$ does not charge $\{z\}$, by Theorem A. If $0<\rho<1$ and $x \in E_{\rho}$, then there exists $x_{0}$ 
in $E$ such that $x \in B\left(x_{0}, \rho d_{K}\left(x_{0}\right)\right)$ and so the volume mean value inequality for superharmonic functions yields

$$
\begin{aligned}
u(x) & \geq l_{n}^{-1}(\rho+\kappa)^{-n}\left\{d_{K}\left(x_{0}\right)\right\}^{-n} \int_{B\left(x,(\rho+\kappa) d_{K}\left(x_{0}\right)\right)} u(y) d y \\
& \geq l_{n}^{-1}(\rho+\kappa)^{-n}\left\{d_{K}\left(x_{0}\right)\right\}^{-n} \int_{B\left(x_{0}, \kappa d_{K}\left(x_{0}\right)\right)} u(y) d y \\
& =(1+\rho / \kappa)^{-n} G_{B}\left(z, x_{0}\right) \\
& \geq \frac{(1-\rho)^{n}}{1-\rho^{2}}(1+\rho / \kappa)^{-n} G_{B}(z, x)=c G_{B}(z, x), \quad \text { say }
\end{aligned}
$$

(see $(2))$. Hence $u / c \geq G_{B}(z, \cdot)$ on $E_{\rho}$, and so

$$
u / c \geq \widehat{R}_{G_{B}(z, \cdot)}^{E_{\rho}} \quad \text { on } B .
$$

Since the Riesz measure associated with $u$ does not charge $\{z\}$, it follows easily that the same is true of the Riesz measure associated with the above reduced function. Thus $E_{\rho}$ is thin at $z$, by Theorem A.

We will also need the result below which follows from a well-known elementary covering lemma (see, for example, [14, pp. 9, 10]).

Lemma 3. Let $K$ be a nonempty compact set and $F$ be a bounded subset of $\mathbb{R}^{n} \backslash K$. Then there is a countable subset $A=\left\{x_{k}: k \geq 1\right\}$ of $F$ such that the balls $\left\{B\left(x_{k}, d_{K}\left(x_{k}\right) / 6\right): k \geq 1\right\}$ are disjoint, and such that $F_{1 / 6} \subseteq A_{5 / 6}$.

It will now be shown that (c) implies (b) in Theorem 1 . Let $\Omega, K, E$ be as in the first sentence of the theorem and suppose that (c) holds. Let $m \in \mathbb{N}$ be large enough so that $B(z, 2 / m) \subseteq \Omega$ for all $z$ in $K$, and let $F=\left\{x \in E: d_{K}(x)<1 / m\right\}$. We choose a countable subset $A=\left\{x_{k}: k \geq 1\right\}$ of $F$ as in Lemma 3. It follows from condition (c) and Lemma 2 that, for any value of $\rho$ in $(0,1)$, the sets $E_{\rho}, F_{\rho}$, and hence $A_{\rho}$, are nonthin at each point of $K$.

Let $f \in C^{+}(K)$ and $0<\varepsilon<1$. We choose $\kappa$ in $(0,1 / 6)$ small enough so that

$$
\begin{aligned}
(1-\varepsilon) G_{\Omega}\left(x_{k}, z\right) \leq G_{\Omega}(x, z) \leq & (1+\varepsilon) G_{\Omega}\left(x_{k}, z\right) \\
& \left(z \in K ; x \in \overline{B\left(x_{k}, \kappa d_{K}\left(x_{k}\right)\right)} ; k \geq 1\right)
\end{aligned}
$$

(see (2)). It follows from Proposition 1 that there is a positive superharmonic function $u$ on $\Omega$ such that $u=f$ on $K$. Let $A(l)=\left\{x \in A: d_{K}(x)>1 / l\right\}$ for each $l$ in $\mathbb{N}$. We take reductions relative to sets in $\Omega$ and observe that

$$
\widehat{R}_{u}^{(A(l))_{\kappa}}(z) \uparrow \widehat{R}_{u}^{A_{\kappa}}(z)=u(z)=f(z) \quad(l \rightarrow \infty ; z \in K)
$$

by the nonthinness of $A_{\kappa}$ at each point $z$ in $K$. This convergence is uniform on $K$ by Dini's theorem, so we can choose $l_{0}$ in $\mathbb{N}$ such that

$$
f(z) \geq \widehat{R}_{u}^{\left(A\left(l_{0}\right)\right)_{\kappa}}(z)>(1-\varepsilon) f(z) \quad(z \in K) .
$$

The reduced function in (4) is the potential of a measure $\nu$ with support contained in $\bigcup_{k \in I} \overline{B\left(x_{k}, \kappa d_{K}\left(x_{k}\right)\right)}$, where $I$ is some finite subset of $\mathbb{N}$. These balls are disjoint, since $\kappa<1 / 6$. If we define $\nu_{k}=\nu\left(\overline{B\left(x_{k}, \kappa d_{K}\left(x_{k}\right)\right)}\right)$ for each $k$ in $I$, then it follows from (3) and (4) that

$$
(1-\varepsilon)^{-1} f(z) \geq \sum_{k \in I} \nu_{k} G_{\Omega}\left(x_{k}, z\right) \geq(1-\varepsilon)(1+\varepsilon)^{-1} f(z) \quad(z \in K) .
$$


We have thus shown that, for any given value of $m$, the function $f$ may be uniformly approximated on $K$ by positive linear combinations of the functions $\left\{G_{\Omega}(x, \cdot): x \in E, d_{K}(x)<1 / m\right\}$. It now follows from Lemma 1 that (b) holds.

4.2. Clearly (b) implies (a), so it remains to prove that (a) implies (c). We again begin with a preparatory lemma. The support of a measure $\mu$ will be denoted by $\operatorname{supp} \mu$. If $\operatorname{supp} \mu$ is a compact subset of a Greenian domain $\Omega$, then we write $G_{\Omega} \mu$ for the potential $\int G_{\Omega}(\cdot, y) d \mu(y)$.

Lemma 4. Let $K$ be a compact set, let $\Omega$ be a Greenian domain which contains $K$, let $z_{0}$ be a limit point of $K$, and let $E \subseteq \Omega \backslash K$. The following are equivalent:

(i) there exists $\rho$ in $(0,1)$ such that $E_{\rho}$ is thin at $z_{0}$;

(ii) there is a measure $\nu$ on $K$ such that $\nu\left(\left\{z_{0}\right\}\right)=0$ and $G_{\Omega} \nu \geq G_{\Omega}\left(z_{0}, \cdot\right)$ on $E$.

To see this, suppose that (ii) holds and let $\rho \in(0,1)$. We observe from (2) that there is a positive constant $c$, depending on $\rho$, such that $c G_{\Omega} \nu \geq G_{\Omega}\left(z_{0}, \cdot\right)$ on $E_{\rho}$. Since $\nu\left(\left\{z_{0}\right\}\right)=0, E_{\rho}$ is thin at $z_{0}$.

Conversely, suppose that (i) holds. Let $a>0$ be such that $\Omega$ contains the set $\left\{x: d_{K}(x)<6 a\right\}$, let $W=\left\{x: d_{K}(x)<a\right\}$, and let $F=E \cap W$. With this choice of $F$, let $A=\left\{x_{k}: k \geq 1\right\}$ be as in Lemma 3 . Then $A_{1 / 12}$ is thin at $z_{0}$, by Lemma 2 . It follows that the Riesz measure $\mu$ associated with the potential $\widehat{R}_{G_{\Omega}\left(z_{0}, \cdot\right)}^{A_{1 / 12}}$ does not charge $\left\{z_{0}\right\}$. Clearly

$$
\operatorname{supp} \mu \subseteq\left(\bigcup_{k} \partial B\left(x_{k}, d_{K}\left(x_{k}\right) / 12\right)\right) \cup K .
$$

For each $k$, where $k \geq 1$, we choose $z_{k}$ in the set $B\left(x_{k}, 2 d_{K}\left(x_{k}\right)\right) \cap\left(K \backslash\left\{z_{0}\right\}\right)$, which is nonempty because $z_{0}$ is not an isolated point of $K$. We now define the measure

$$
\nu=\left.\mu\right|_{K}+\sum_{k} \mu\left(\partial B\left(x_{k}, d_{K}\left(x_{k}\right) / 12\right)\right) \delta_{z_{k}},
$$

where $\delta_{x}$ denotes the unit measure concentrated on $\{x\}$. Clearly $\nu\left(\left\{z_{0}\right\}\right)=0$. Next we claim that there exists $c$ in $(0,1)$ such that

$$
G_{\Omega}\left(z_{k}, x_{j}\right) \geq c G_{\Omega}\left(x, x_{j}\right) \quad\left(x \in \partial B\left(x_{k}, d_{K}\left(x_{k}\right) / 12\right) ; j \geq 1 ; k \geq 1\right) .
$$

To see this, we note from Harnack's inequalities that there is a positive constant $c_{1}$ such that

$$
G_{\Omega}\left(z_{k}, x_{k}\right) \geq c_{1} G_{\Omega}\left(x, x_{k}\right) \quad\left(x \in \partial B\left(x_{k}, d_{K}\left(x_{k}\right) / 12\right) ; k \geq 1\right) .
$$

Similarly, there is a positive constant $c_{2}$ such that

$$
\begin{gathered}
G_{\Omega}\left(z_{k}, y\right) \geq c_{2} G_{\Omega}(x, y) \\
\left(x \in \partial B\left(x_{k}, d_{K}\left(x_{k}\right) / 12\right) ; y \in \Omega \backslash B\left(x_{k}, 3 d_{K}\left(x_{k}\right)\right) ; k \geq 1\right) .
\end{gathered}
$$

If $j \neq k$ and $x_{j} \in B\left(x_{k}, 3 d_{K}\left(x_{k}\right)\right)$, then $x_{j} \notin B\left(x_{k}, d_{K}\left(x_{k}\right) / 6\right)$ by our choice of $A$. It follows from the minimum principle and Harnack's inequalities that there is a positive constant $c_{3}$, independent of the particular values of $j$ and $k$, such that

$$
\begin{aligned}
G_{\Omega}\left(z_{k}, x_{j}\right) & \geq \inf _{\partial B\left(x_{k}, 4 d_{K}\left(x_{k}\right)\right)} G_{\Omega}\left(\cdot, x_{j}\right) \\
& \geq c_{3} G_{\Omega}\left(x, x_{j}\right) \quad\left(x \in \partial B\left(x_{k}, d_{K}\left(x_{k}\right) / 12\right)\right) .
\end{aligned}
$$

If we combine (6), (7), and (8), we obtain (5) and hence

$$
G_{\Omega} \nu\left(x_{j}\right) \geq c G_{\Omega} \mu\left(x_{j}\right)=c G_{\Omega}\left(z_{0}, x_{j}\right) \quad(j \geq 1) .
$$


It follows from (2) that there is a positive constant $c^{\prime}$ such that $G_{\Omega} \nu \geq c^{\prime} G_{\Omega}\left(z_{0}, \cdot\right)$ on $A_{5 / 6}$, which contains $F$. Harnack's inequalities also show that the set

$$
\left\{G_{\Omega}\left(z_{0}, x\right) / G_{\Omega}(z, x): x \in \Omega \backslash W \text { and } z \in K\right\}
$$

is bounded, so $G_{\Omega} \nu / G_{\Omega}\left(z_{0}, \cdot\right)$ has a positive lower bound on all of $E$. Thus, if we multiply $\nu$ by a suitable positive constant, we obtain (ii). This completes the proof of Lemma 4.

We will now show that (a) implies (c) in Theorem 1. Suppose that condition (a) holds and let $z_{0} \in K$.

We deal first with the case where $z_{0}$ is an isolated point of $K$; that is, there is a positive number $\eta$ such that $B\left(z_{0}, \eta\right) \cap K=\left\{z_{0}\right\}$. Suppose that $z_{0} \notin \bar{E}$. Since $K$ has more than one point, we can define

$$
a=\sup \left\{G_{\Omega}\left(x, z_{0}\right) / G_{\Omega}(x, z): x \in E \text { and } z \in K \backslash\left\{z_{0}\right\}\right\} .
$$

To see that $a$ is finite, let $W$ be a bounded open set such that $K \subset W$ and $\bar{W} \subset \Omega$. As before, it follows from Harnack's inequalities that the set

$$
\left\{G_{\Omega}\left(x, z_{0}\right) / G_{\Omega}(x, z): x \in \Omega \backslash W \text { and } z \in K\right\}
$$

is bounded. Also, $G_{\Omega}\left(\cdot, z_{0}\right)$ is bounded above on $W \cap E$, since $z_{0} \notin \bar{E}$, while $G_{\Omega}(\cdot, \cdot)$ has a positive lower bound on $(W \cap E) \times K$. Hence $a<+\infty$. We now define

$$
f(z)= \begin{cases}a+1 & \left(z=z_{0}\right), \\ 1 & \left(z \in K \backslash\left\{z_{0}\right\}\right),\end{cases}
$$

whence $f \in C^{+}(K)$. However, if (1) holds, then

$$
\begin{aligned}
f\left(z_{0}\right) & =\sum_{k} \lambda_{k} G_{\Omega}\left(x_{k}, z_{0}\right) \leq a \sum_{k} \lambda_{k} G_{\Omega}\left(x_{k}, z\right) \\
& =a f(z)=a \quad\left(z \in K \backslash\left\{z_{0}\right\}\right),
\end{aligned}
$$

which yields a contradiction. Therefore $z_{0} \in \bar{E}$. Since $z_{0}$ is an isolated point of $K$, it follows that $E_{\rho}$ is nonthin at $z_{0}$ for any $\rho$ in $(0,1)$.

It remains to consider the case where $z_{0}$ is a limit point of $K$. Let $\rho \in(0,1)$ and suppose that $E_{\rho}$ is thin at a point $z_{0}$ in $K$. It follows from Lemma 4 that there is a measure $\nu$ on $K$ such that $\nu\left(\left\{z_{0}\right\}\right)=0$ and $G_{\Omega} \nu \geq G_{\Omega}\left(z_{0}, \cdot\right)$ on $E$. Let $x \in \Omega \backslash K$. Then $G_{\Omega}(x, \cdot) \in C^{+}(K)$ so, by hypothesis, there exist a sequence $\left(x_{k}\right)$ of points in $E$ and a sequence $\left(\lambda_{k}\right)$ of positive numbers such that

$$
G_{\Omega}(x, z)=\sum_{k} \lambda_{k} G_{\Omega}\left(x_{k}, z\right) \quad(z \in K) .
$$

If we integrate with respect to $d \nu(z)$, we see by monotone convergence that

$$
G_{\Omega} \nu(x)=\sum_{k} \lambda_{k} G_{\Omega} \nu\left(x_{k}\right) \geq \sum_{k} \lambda_{k} G_{\Omega}\left(x_{k}, z_{0}\right)=G_{\Omega}\left(x, z_{0}\right) .
$$

Thus $G_{\Omega} \nu \geq G_{\Omega}\left(z_{0}, \cdot\right)$ on all of $\Omega \backslash K$. Since $\nu\left(\left\{z_{0}\right\}\right)=0$, we obtain the contradictory conclusion that $\Omega \backslash K$ (and hence also $\Omega$ ) is thin at $z_{0}$. Therefore $E_{\rho}$ is nonthin at $z_{0}$.

This completes the proof of Theorem 1. 


\section{Proof of Corollary 1}

Let $1 \leq m \leq n-3$ and let $K$ be a compact $m$-dimensional Lipschitz manifold in $\mathbb{R}^{n}$. Further, let $\left\{Q_{k}: k \in \mathbb{N}\right\}$ be a Whitney cube decomposition (see, for example, $\left[14\right.$, p. 16]) of $\mathbb{R}^{n} \backslash K$, let $F$ be a bounded set in $\mathbb{R}^{n}$, and let

$$
\widetilde{F}=\bigcup_{\left\{k: Q_{k} \cap F \neq \varnothing\right\}} Q_{k} .
$$

We record below special cases of two results of Aikawa; see [1, Corollaries 4 and 5] and the first paragraph on p. 16 of that paper.

Theorem B. Suppose that $F$ is Borel measurable. If $F$ is thin at a point $z$ in $K$, then

$$
\int_{F} d_{K}(x)^{-2}|x-z|^{2-n} d x<+\infty .
$$

Theorem C. Let $z \in K$. The following are equivalent:

(a) $\widetilde{F}$ is thin at $z$;

(b) $\int_{\widetilde{F}} d_{K}(x)^{-2}|x-z|^{2-n} d x<+\infty$.

Let $E$ be any subset of $\mathbb{R}^{n} \backslash K$ and let $F=\left\{x \in E: d_{K}(x)<1\right\}$. An argument directly analogous to the proof of Lemma 2 shows that, if $\widetilde{F}$ is thin at a point $z$ of $K$, then $F_{\rho}$ is thin at $z$ for every $\rho$ in $(0,1)$. Now suppose that there exists $\rho$ in $(0,1)$ such that $E_{\rho}$ (and hence $F_{\rho}$ ) is nonthin at each point of $K$. Then the same is true of $\widetilde{F}$, and it follows easily from Theorem $\mathrm{C}$ that condition (b) of Corollary 1 holds (any value of $\rho$ in $(0,1)$ will do). Conversely, if condition (b) of Corollary 1 holds, then $E_{\rho} \cap\left\{x: d_{K}(x)<1\right\}$ is nonthin at each point of $K$, by Theorem B, and the same is therefore true of $E_{\rho}$. It is now clear that Corollary 1 follows from Theorem 1.

Aikawa's results do not cover the case where $m=n-2$. We do not know what can be said in this case.

\section{REFERENCES}

1. H. Aikawa, Quasiadditivity of Riesz capacity Math. Scand. 69 (1991), 15-30. MR 93d:31007

2. - Sets of determination for harmonic functions in an NTA domain preprint.

3. F. F. Bonsall and D. Walsh, Vanishing $l^{1}$-sums of the Poisson kernel, and sums with positive coefficients, Proc. Edinburgh Math. Soc. 32 (1989), 431-447. MR 90m:31001

4. M. Brelot, Sur l'approximation et la convergence dans la théorie des fonctions harmoniques ou holomorphes, Bull. Soc. Math. France 73 (1945), 55-70. MR 7:205a

5. J. Deny, Sur l'approximation des fonctions harmoniques, Bull. Soc. Math. France 73 (1945), 71-73. MR 7:205b

6. J. L. Doob, Classical potential theory and its probabilistic counterpart, Springer, Berlin, 1984. MR 85k:31001

7. N. F. Dudley Ward, On a decomposition theorem for continuous functions of Hayman and Lyons, New Zealand J. Math. 22 (1993), 49-59.

8. M. Essén, On minimal thinness, reduced functions and Green potentials, Proc. Edinburgh Math. Soc. 36 (1992), 87-106. MR 94c:31003

9. S. J. Gardiner, Sets of determination for harmonic functions, Trans. Amer. Math. Soc. 338 (1993), 233-243. MR 93j:31005

10. W. K. Hayman, Atomic decompositions, Recent Advances in Fourier Analysis and its Applications (J. S. Byrnes and J. L. Byrnes, eds.), Kluwer, Dordrecht, 1993, pp. 597-611.

11. W. K. Hayman and T. J. Lyons, Bases for positive continuous functions, J. London Math. Soc. (2) 42 (1990), 292-308. MR 92a:31002 
12. M. V. Keldyš, On the solvability and stability of the Dirichlet problem, Uspekhi Mat. Nauk 8 (1941), 171-231; English transl., Amer. Math. Soc. Transl. 51 (1966), 1-73.

13. N. S. Landkof, Foundations of modern potential theory, Springer, Berlin, 1972. MR 50:2520 14. E. M. Stein, Singular integrals and differentiability properties of functions, Princeton Univ. Press, Princeton, NJ, 1970. MR 44:7280

15. H. Wallin, Continuous functions and potential theory, Ark. Mat. 5 (1963), 55-84. MR 29:2425

Department of Mathematics, University College, Dublin 4, Ireland

E-mail address: gardiner@irlearn.ucd.ie 